\title{
Unstable flux flow due to heated electrons in $\mathrm{Y}-\mathrm{Ba}-\mathrm{Cu}-\mathrm{O}$ films
}

\author{
Milind N. Kunchur \\ Department of Physics and Astronomy \\ University of South Carolina, Columbia, SC 29208
}

(Dated: Accepted August 5, 2002)

\begin{abstract}
A flux instability occurs in superconductors at low temperatures, where ee scattering is more rapid than $e p$, whereby the dissipation significantly elevates the electronic temperature while maintaining a thermal-like distribution function. The reduction in condensate and rise in resistivity produce a non-monotonic current-voltage response. In contrast to the Larkin-Ovchinnikov instability where the vortex shrinks, in this scenario the vortex expands and the quasiparticle population rises. Measurements in $\mathrm{Y}_{1} \mathrm{Ba}_{2} \mathrm{Cu}_{3} \mathrm{O}_{7-\delta}$ agree quantitatively with the distinct predictions of this mechanism.
\end{abstract}

PACS numbers: 74.60.Ge, 74.72.Bk, 71.10.Ca, 71.38.-k, 72.10.Di, 72.15.Lh, 73.50.Fq

Keywords: Flux, fluxon, vortex, superconductivity, critical, current, instability, unstable, YBCO

In a type II superconductor, magnetic fields between the lower critical value $H_{c 1}$ and upper critical field $H_{c 2}$ introduce flux vortices containing a quantum of flux $\Phi_{o}=h / 2 e$. Here we have superconducting films in a perpendicular applied flux density $B$, with a transport electric current density $j$ in the plane of the film, which exerts a Lorentz driving force $F_{L}=j \Phi_{o}$. The vortex motion generates an electric field $E=v B$ and is opposed by a viscous drag $\eta v$ ( $\eta$ is the coefficient of viscosity and $v$ the vortex velocity), so that in steady state $j \Phi_{o}=\eta v$ and the response is Ohmic. Larkin and Ovchinnikov (1986) have shown that a dirty superconductor at low temperatures has a free-flux-flow resistivity related to the normalstate value $\rho_{n}$ by [1]

$$
\rho_{f} / \rho_{n} \simeq 0.9 B / H_{c 2}(T) .
$$

Approximately the same result, without the precise 0.9 prefactor, can be obtained by considering the Ohmic dissipation in the core and temporal changes in the order parameter leading to irreversible entropy transfer [2]; the result is also valid for $d$-wave superconductors that are not superclean 3. Eq. 11 is equivalent to $\eta \approx \Phi_{0} H_{c 2} / \rho_{n}$. At low levels of $j$ and $E$, in the assumed dirty limit $l \ll \xi E_{F} / k T_{c}$ ( $l$ is the mean free path and $E_{F}$ is the Fermi energy), $\eta$ is a constant that is proportional to the order parameter $\Delta$ and inversely proportional to the size $\sim \xi^{2}$ (where $\xi$ is the coherence length) of the vortex [ $⿴$. (The Hall effect and transverse component of $E$ are negligible for this discussion, as is vortex pinning because of the large driving forces [5].)

At high electric fields and dissipation levels sufficient to alter the electronic distribution function and/or the electronic temperature, $j(E)$ becomes non-linear and can develop an unstable region $(d j / d E<0)$ above some critical vortex velocity $v^{*}$. For the regime near $T_{c}$, such an instability has been predicted by Larkin and Ovchinnikov (LO) $\sqrt{6}$, and has been experimentally well established [7, 8, 9, 10]. At high temperatures, the electron-phonon $(e p)$ scattering time $\tau_{e p}$ can be shorter than the electronelectron (ee) scattering time $\tau_{e e}$, preventing internal equi- libration of the electronic system and producing a peculiar non-thermal distribution function. Since the order parameter $\Delta$ is especially sensitive to the distribution function when close to $T_{c}$, even moderate values of $E$ can sufficiently distort $\Delta$ (via the Eliashberg mechanism [11]) causing a shrinkage of the vortex core and a removal of quasiparticles from its vicinity. This is the gist of the LO behavior [6, 7. As LO themselves emphasize, the effect is most favorable close to $T_{c}$ for superconductors with a full gap and, as shown by Bezuglyj and Shklovskij [12], is dominant for $B<B_{T}$ (with $B_{T} \sim 0.1 \mathrm{~T}$ for our low- $T$ regime). One of the predictions of the standard LO effect is a $v^{*}$ that is $B$ independent. This has been confirmed in $\mathrm{Y}_{1} \mathrm{Ba}_{2} \mathrm{Cu}_{3} \mathrm{O}_{7-\delta}$ in the high- $T$ range [9, 10].

This work investigates the opposite regime of $T \ll T_{c}$ and $B>B_{T}$, where $\Delta$ is not sensitive to small changes in the distribution function. Furthermore because $\tau_{e e}<$ $\tau_{e p}$ as $T \rightarrow 0$, the distribution function remains thermal like and the electronic system suffers mainly a temperature shift with respect to the lattice [6, 12, 13, 14]. Then instead of the standard LO picture described earlier, we consider a more transparent scenario where the main effect of the dissipation is to raise the electronic temperature, create additional quasiparticles, and diminish $\Delta$. The vortex expands rather than shrinks, and the viscous drag is reduced because of a softening of gradients of the vortex profile rather than a removal of quasiparticles. This sequence of events is almost opposite to the standard LO picture and represents a new type of unstable regime prevalent at $T \ll T_{c}$. All experimental measurables can be calculated without ambiguity, and the predicted field dependencies and full $j(E)$ curves fit the experimental results without any adjustable parameters.

Previously some deviations from LO behavior at intermediate temperatures - such as a $B$-dependent $v^{*}$-were treated through modifications to the LO effect, such as an intervortex spacing $l_{\phi}$ that exceeds the energy-relaxation length $l_{\epsilon}$ [15] or by inclusion of thermal effects [12, 13. Those treatments do not apply to the present $T \ll T_{c}$ regime where $l_{\epsilon} \sim \sqrt{D \tau_{e p}} \sim 100-1000 \mathrm{~nm}$ is larger than 
$l_{\phi}=1.075 \sqrt{\Phi_{o} / B} \sim 10-50 \mathrm{~nm}\left(D=3 \times 10^{-4} \mathrm{~m}^{2} / \mathrm{s}\right.$ is the diffusion constant [9]).

Here we take a "bottom-up" approach and start from the $T \sim 0$ limit: The total input power $j E$ travels from electrons to lattice and from there to the bath, so that $T_{0}<T_{p}<T^{\prime}$, where $T_{0}$ and $T_{p}$ are the bath and phonon temperatures, and $T^{\prime}$ is the raised non-equilibrium electronic temperature. Macroscopic heating, represented by $T_{p}-T_{0}=R_{t h} j E$, is $<5 \%$ of the total increase $T^{\prime}-T_{0}$ for the worst case dissipation so that $T_{p} \approx T_{0}$ (Here $R_{t h} \sim 1$ $\mathrm{nK} . \mathrm{cm}^{3} / \mathrm{W}$ is the total thermal resistance between the film and the bath; see experimental section. It will be seen later that the specific-heat integral heavily weights the higher temperatures, so that $T_{p^{-}} T_{0}$ is quite negligible.). The energy relaxation between electrons and lattice occurs by inelastic $e p$ scattering, and is characterized by an effective time $\left.\tau_{\epsilon} \sim<\tau_{e p}\right\rangle$. The principle contributions to $\rho_{n}$ come from impurities and phonons. Since the phonon temperature remains near the bath, $\rho_{n}$ 's value will not change as the non-equilibrium $T^{\prime}$ rises. Thus putting $\rho_{n}\left(T_{0}\right)$ and $H_{c 2}\left(T^{\prime}\right)$ (since $H_{c 2}$ does depend on $\left.T^{\prime}\right)$ into previous equations gives the $j(E)$ response in terms of $T^{\prime}$ :

$$
j=v \eta\left(T^{\prime}\right) / \Phi_{0}=E H_{c 2}\left(T^{\prime}\right) / \rho_{n}\left(T_{0}\right) B .
$$

One now has the ingredients for calculating the critical field dependencies in a few steps. The $j(E)$ function of Eq. 22 is non-monotonic since $E$ is multiplied by $H_{c 2}\left(T^{\prime}\right)$ (or $\eta\left(T^{\prime}\right)$ ) which drops rapidly to zero as $T^{\prime} \rightarrow T_{c}$ with increasing dissipation. The instability occurs at $d j / d E=0$, which happens at a certain value $T^{\prime}=T^{*}$ where $H_{c 2}\left(T^{\prime}\right)$ (or $\eta\left(T^{\prime}\right)$ ) drops sufficiently rapidly. $T^{\prime}$ depends explicitly only on the power density $j E=\eta v^{2} B / \Phi_{0}$ and on quantities that depend on $T^{\prime}$ itself $\left(\tau_{\epsilon}\right.$, specific heat, etc.). Hence at the instability, $j^{*} E^{*}=v^{* 2} \eta\left(T^{*}\right) B / \Phi_{0}=$ constant, which gives the critical-parameter field dependencies:

$$
v^{*} \propto 1 / \sqrt{B}, E^{*} \propto \sqrt{B}, j^{*} \propto 1 / \sqrt{B}, \text { and } \rho^{*} \propto B
$$

using $E^{*}=v^{*} B, j^{*}=v^{*} \eta\left(T^{*}\right) / \Phi_{0}$, and $\rho^{*}=E^{*} / j^{*}$. This gives $v^{*} \propto 1 / \sqrt{B}$ in a natural way, consistent with our measurements in this regime and in contrast to the $B$ independent $v^{*}$ of the pure LO effect near $T_{c}$.

To derive the complete $j(E)$ response and absolute values of critical parameters we calculate $T^{\prime}$ and insert it into Eq. 2. We take $H_{c 2}(0)=120 \mathrm{~T}$ [16] with the WHH function [17] for interpolation between this $H_{c 2}(0)=120$ and $H_{c 2}\left(\overline{T_{c}}\right)=0$ (There is some theoretical controversy regarding the exact form of $H_{c 2}(T)$; however, empirically, direct measurements [16] of $H_{c 2}(T)$, within their uncertainity, seem not to depart drastically from the WHH function, and the exact functional shape does not crucially affect our conclusions.). The dissipation raises the electronic energy by $j E \tau_{\epsilon}$, which is related to $T^{\prime}$ by

$$
j E \tau_{\epsilon} \approx \frac{E^{2} H_{c 2}\left(T^{\prime}\right) \tau_{\epsilon}}{\rho_{n}\left(T_{0}\right) B} \approx \int_{T_{p}}^{T^{\prime}} c(T) d T \approx \int_{T_{0}}^{T^{\prime}} c(T) d T
$$

where $c(T)$ is the electronic specific heat. To calculate $c(T), \mathrm{Y}_{1} \mathrm{Ba}_{2} \mathrm{Cu}_{3} \mathrm{O}_{7-\delta}$ is modeled as a layered $d$ wave superconductor: $\Delta_{\vec{k}}(T)=\Delta_{0}(T)\left[k_{x}^{2}-k_{y}^{2}\right] / k^{2} \approx$ $\Delta_{0}(T) \cos (2 \theta)$, taking the BCS temperature dependence for $\Delta_{0}(T)$ and $\Delta_{0}(0)=19 \mathrm{meV}$ from tunneling and infrared measurements [18]. Then $c(T)=\partial\left\{\sum_{k} E_{k} f_{k}\right\} / \partial T$, where $f_{k}=\left[\exp \left(E_{k} / k_{B} T\right)+1\right]^{-1}$ is the Fermi-Dirac distribution, $E_{k}=\sqrt{\zeta_{k}^{2}+\Delta_{k}^{2}}, \zeta_{k}=\epsilon_{k}-\mu$, and $\mu=0.2 \mathrm{eV}$ is the chemical potential [19]. With the replacement of $\sum_{k}$ by $\int d \zeta N(0) \int d \theta / 2 \pi$ (where $N(0)$ is the normal density

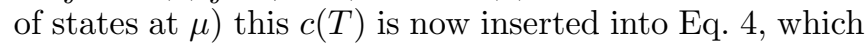
is integrated numerically to obtain $T^{\prime}$ and thence $j(E)$ from Eq. 2 .

The numerical results of the above calculation are shown in Fig. 1. The $j(E)$ curves of panel (a) have an infinite slope at the instability $\left(j^{*}, E^{*}\right)$, indicated by arrows, and then exhibit negative slope. This negative sloped portion is experimentally forbidden in a current biased measurement and instead will be manifested as a vertical jump in $E$. The electronic temperature $T^{\prime}$ rises from the bath value $T_{0}$ to $T^{*}$ at the instability. Panel (b) shows the computed $T^{*}$, which is independent of $B$ as expected but has a slight dependence on the bath temperature $T_{0}$.
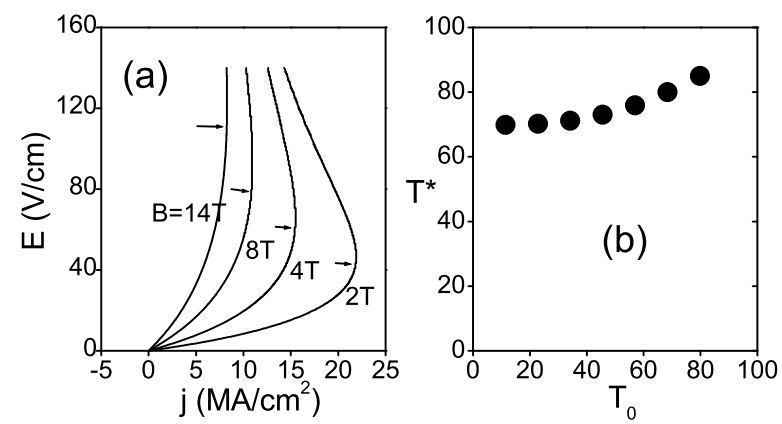

FIG. 1: Numerical results obtained from solving Eq. 目, as described in the text. (a) Theoretical $j(E)$ curves, at $T_{0}=$ 0K. The onset of negative slope, indicated by arrows, marks the instabilities. (b) The critical temperature $T^{*}$ for different initial temperatures $T_{0} ; T^{*}$ is independent of $B$ and $\tau_{\epsilon}$.

In order to conveniently scale the experimental curves, the exact numerical $j(E)$ function derived above - and plotted in Fig. 1 can be cast into a mathematically more manageable form by noting that the R.H.S. of Eq. 1 can be approximated [? ] by $j E \tau_{\epsilon} \approx \Delta\left(T^{\prime}\right) n_{q}\left(T^{\prime}\right)-$ $\Delta\left(T_{p}\right) n_{q}\left(T_{p}\right) \approx \Delta\left(T^{\prime}\right) n_{q}\left(T^{\prime}\right) . \Delta$ and $n_{q}$ are connected through the statistical equations of the previous paragraph. Numerically computing the number of quasiparticles excited above the gap (taking the anisotropic $d$-wave 
gap together with a BCS temperature dependence as discussed above) we obtain the following $d$-wave generalization of the $\Delta-n_{q}$ relationship [? ]: $\left(\Delta / \Delta_{0}\right)^{2}=f\left(n_{q} / n\right)$, where $n=2.7 \times 10^{21} \mathrm{~cm}^{-3}$ is the carrier concentration and $f(x) \simeq 1-0.4386 x-1539 x^{2}+40381 x^{3}-345217 x^{4}$ (despite the appearance of successively increasing coefficients in $f(x)$, the terms rapidly converge because $\left.x=n_{q} / \mathrm{n} \sim k_{B} T / E_{F} \sim 0.01\right)$. Combining this with the earlier $j E \tau_{\epsilon} \approx \Delta\left(T^{\prime}\right) n_{q}\left(T^{\prime}\right), \eta=j \Phi_{0} / v=j \Phi_{0} B / E$, $\eta \approx H_{c 2} \Phi_{0} / \rho_{n}$, and $\eta \propto \Delta$, we get a convenient closed form for the non-linear $j(E)$ characteristic:

$$
j \approx\left(\frac{H_{c 2}\left(T_{0}\right)}{B \rho_{n}\left(T_{0}\right)}\right) E \sqrt{f(x)}
$$

with $x=n_{q} / n=0.0245 \times E^{2} / E^{* 2}$, and $E^{*}=$ $\sqrt{0.0245 \rho_{n}\left(T_{0}\right) B n \Delta_{0} / \tau_{\epsilon} H_{c 2}\left(T_{0}\right)}$ is the value of $E$ at the instability peak. We now turn to the experimental details and results.

The samples are $c$-axis oriented epitaxial films of $\mathrm{Y}_{1} \mathrm{Ba}_{2} \mathrm{Cu}_{3} \mathrm{O}_{7-\delta}$ on (100) $\mathrm{LaAlO}_{3}$ substrates with $T_{c}$ 's around $90 \mathrm{~K}$ and of thickness $t \approx 90 \mathrm{~nm}$. Electron-beam lithography was used to pattern bridges of widths $w \approx$ 2-20 $\mu \mathrm{m}$ and lengths $l \approx 30-200 \mu \mathrm{m}$. Altogether ten samples were studied at 12 temperatures $(1.6,2.2,6,7$, $8,10,20,27,35,42,50,80 \mathrm{~K}$ ) and at 13 flux densities $(0.1,0.2,0.5,1,1.5,2,10,11,13,13.5,13.8,14,15.8 \mathrm{~T})$. The electrical transport measurements were made with a pulsed constant current source, preamplifier circuitry, and a digital storage oscilloscope. The pulse rise times are about $100 \mathrm{~ns}$ with a duty cycle of about $1 \mathrm{ppm}$, which for the narrowest bridges result in effective thermal resistances of order $1 \mathrm{nK} . \mathrm{cm}^{3} / \mathrm{W}$. Note that the $j$ values in the experiment are an order of magnitude lower than the depairing current density [20] and the applied flux densities exceed the self field of the current by at least two orders of magnitude. Further details about the experimental techniques are discussed elsewhere [21].

Fig. 2(a) shows a typical set of experimental $j(E)$ curves. The last stable datapoint $\left(j^{*}, E^{*}\right)$ of each curve is at the tail of each arrow. The slightest further increase of $j>j^{*}$ causes a drastic vertical jump in $E$ as shown by the arrows (the voltage pulse jumps off the scale of the oscilloscope upto the compliance limit of the current source). The jumps show only a small hysteresis $<3 \%$ of $j^{*}$. (As expected for a current-biased measurement, the break occurs slightly before the slope has become quite vertical [12].) Fig. 2(b) shows the same data plotted as the R.H.S. of Eq. 5 vs $j-j_{c 0}$, the excess current density over the depinning value. The data scale well and tend toward homogeneous linearity. Note that the collapse implies an excellent proportionality between $\rho$ and $B$ over the entire range.

Fig. 33 shows experimentally measured $B$ dependencies of $v^{*}$ and $\rho^{*}$ for $T_{0}=1.6 \mathrm{~K}$, demonstrating excellent agreement with Eq. 3 (the other dependencies $E^{*} \propto \sqrt{B}$ and
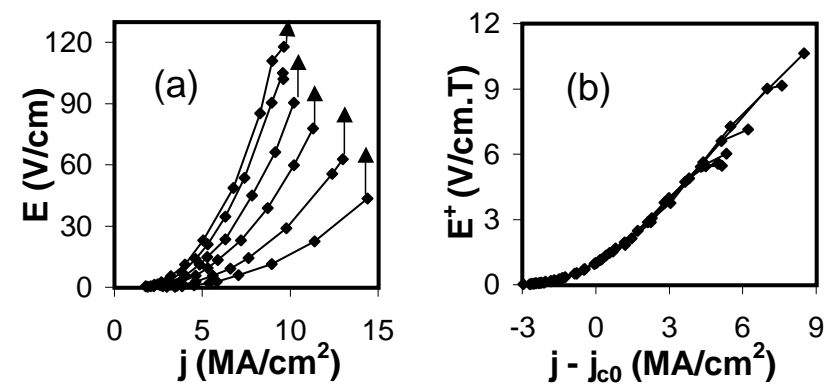

FIG. 2: Experimental current-voltage characteristics. (a) Raw values of $E$ versus $j$ at $T=20 \mathrm{~K}$ and applied $B$ values of (from lowest to highest curve) $3,5,8,11,13.8$, and 15.8 $\mathrm{T}$. The last symbol on each curve is right at the instability. Slightest further increase of $j$ (entering the forbidden negative-sloped portion of the theoretical curves of Fig. 1) causes $E$ to make discontinous vertical jumps (arrows). (b) The same data plotted as $E^{+}=(E / B) \times \sqrt{f\left(0.0245 E^{2} / E^{* 2}\right)}$ (as per Eq. 1) versus $j-j_{c 0}$, where the critical depinning current density $j_{c 0}$ is defined at $E^{+}=1 \mathrm{~V} / \mathrm{cm}$. T (the scaling is not affected by the choice of criterion).
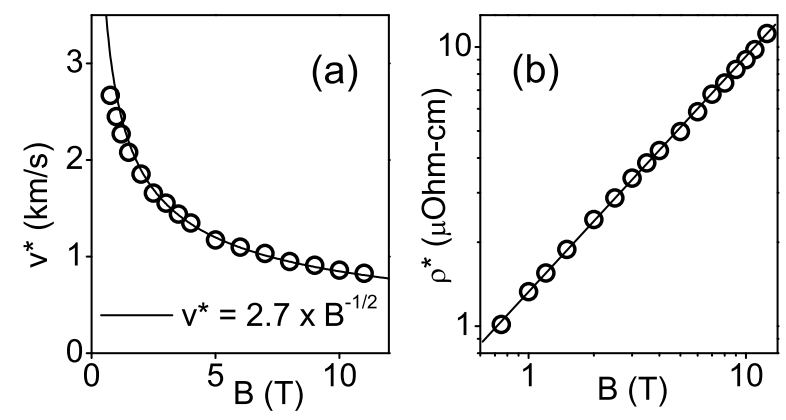

FIG. 3: Variation of critical parameters with flux density. The measurements were made at $T=1.6 \mathrm{~K}$. (a) The critical velocity shows a $v^{*} \propto 1 / \sqrt{B}$ trend (solid line is a $1 / \sqrt{B}$ fit). (b) The critical resistivity is proportional to the flux density (straight line is a guide to the eye).

$j^{*} \propto 1 / \sqrt{B}$ follow from $\rho^{*} \propto B$ and $\left.v^{*} \propto 1 / \sqrt{B}\right)$. The $v^{*} \propto 1 / \sqrt{B}$ dependence was found to be ubiquitous for all of our low- $T$ measurements in ten samples (spanning $1.6 \mathrm{~K} \leq T \leq 50 \mathrm{~K}$ and $0.5 \mathrm{~T} \leq B \leq 15.8 \mathrm{~T}$ ) and has also been seen by Xiao et al. 10] at intermediate temperatures (at the lower end of their $T \sim 60-90 \mathrm{~K}$ range of study). Note that the excellent linearity between between $\rho^{*}$ and $B$ demonstrates the independence of $\eta$ on $B$ in this regime; then the resistivity is simply proportional to the number of vortices and hence $B$.

The final step in the analysis is to extract $\tau_{\epsilon}$ from the data. Inverting Eq. A evaluated at the instability (i.e., at $T^{\prime}=T^{*}$ ), we get $\tau_{\epsilon} \approx \frac{\rho_{n}\left(T_{0}\right) B}{H_{c 2}\left(T^{*}\right) E^{* 2}} \int_{T_{0}}^{T^{*}} c(T) d T$; the $T^{*}\left(T_{0}\right)$ function comes from the model itself (Fig. 1 1 (b)). $\tau_{\epsilon}$ calculated in this way is shown by the circles in Fig. 6 . If the data are analyzed in the LO frame- 
work $\left(\tau_{\epsilon} \approx D[14 \zeta(3)]^{1 / 2} B^{2} /\left(\pi E^{* 2}\right)\right)$ a markedly longer $\tau_{\epsilon}$ is obtained shown by the squares. For comparison the arrow shows the theoretical $T \rightarrow 0$ limiting value estimated from spontaneous emission of phonons. (Emission of non-equilibrium phonons dominates $e p$ scattering by thermal phonons below a cross-over temperature $T_{x}=m v_{F} c_{s} / k_{B} \approx 43 \mathrm{~K}$ producing an essentially $T$-independent scattering rate (since $k T \ll E_{F}$ ) of $\tau_{e p}=\left(3 \pi \rho_{M} c_{s} \hbar^{2}\right) /\left(2 C^{2} m k_{F}\right) \approx 8.4 \times 10^{-11}$, where $C \approx 6 \times$ $10^{-13}$ ergs is the coupling between electron energy and crystal dilation, $c_{s}=4 \mathrm{~km} / \mathrm{s}$ is the sound velocity, and $\rho_{M}=6 \mathrm{~g} / \mathrm{cm}^{3}$ is the mass density 22] for further discussion see, for example, Kittel [23].)

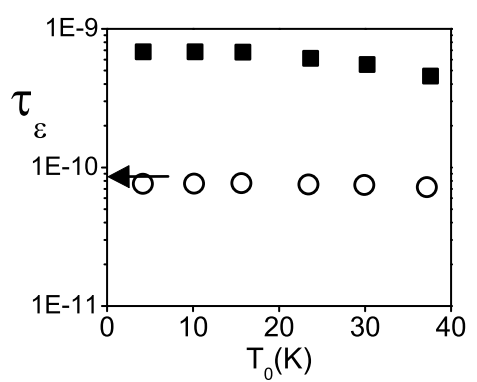

FIG. 4: The energy relaxation time $\tau_{\epsilon}$ extracted from the measured data using the present low-temperature instability model (circles) and from LO theory (squares). The leftward arrow represents the theoretical $T \rightarrow 0$ limiting value of $\tau_{e p}$ from phonon emission (please see text).

In conclusion, we investigated the low-temperature regime of flux motion driven far beyond free flux flow, and observed an instability under all conditions of fields, and temperatures from $\sim T_{c} / 2$ down to essentially $T \approx 0$. The nature of this low-temperature instability seems to be well described by a model where the electron gas is heated above the phonon temperature leading to the generation of quasiparticles and loss in viscosity as the vortex core expands and $\Delta$ is reduced. This scenario is different from the standard LO picture (dominant mainly near $T_{c}$ ) where the vortex shrinks and quasiparticles leave its vicinity. Because the present effect prevails even at temperatures well below $T_{c}$ (where most superconductive devices operate) it becomes an important consideration in the design of applications where the superconductor operates in the dissipative regime 24, since the instability triggers an abrupt rise in dissipation at $j$ values much below the depairing current density. Detailed predictions of the model, including field dependencies of critical parameters and shapes of $j(E)$ curves were experimentally verified, and the effect provides an estimate of the $T \rightarrow 0$ time scale for energy exchange between quasiparticles and phonons.

The author gratefully acknowledges useful discussions and other assistance from J. M. Knight, B. I. Ivlev, M.
Geller, D. K. Christen, J. M. Phillips, R. P. Huebener, N. Schopohl, J. Blatter, and V. Geshkenbein. This work was supported by the U. S. Department of Energy through grant number DE-FG02-99ER45763.

URL: http://www.physics.sc.edu/kunchur; Electronic address: kunchur@sc.edu

[1] A. I. Larkin and Yu. N. Ovchinnikov, in Nonequilibrium Superconductivity, D. N. Langenberg and A. I. Larkin, eds. (Elsevier, Amsterdam, 1986), Ch. 11.

[2] J. Bardeen and M. J. Stephen, Phys. Rev. 140, A1197 (1965); M. Tinkham, Phys. Rev. Lett. 13, 804 (1964); and J. R. Clem, Phys. Rev. Lett. 20, 735 (1968).

[3] N. B. Kopnin and G. E. Volovik, Phys. Rev. Lett. 79, 1377 (1997).

[4] A physically intuitive discussion can be found in Michael Tinkham, Introduction to Superconductivity, 2nd Edition (McGraw Hill, New York, 1996).

[5] M. N. Kunchur, D. K. Christen, and J. M. Phillips, Phys. Rev. Lett. 70, 998 (1993).

[6] A. I. Larkin and Yu. N. Ovchinnikov, Zh. Eksp. Teor. Fiz. 68, 1915 (1975) [Sov. Phys. JETP 41, 960 (1976)].

[7] W. Klein, R. P. Huebener, S. Gauss, and J. Parisi, J. Low Temp. Phys. 61, 413 (1985).

[8] L. E. Musienko, I. M. Dmitrenko, and V. G. Volotskaya, Pis'ma Zh. Eksp.Teor. Fiz. 31, 603 (1980) [JETP Lett. 31, 567 (1980)]; and A. V. Samoilov et al., Phys. Rev. Lett. 75, 4118 (1995).

[9] S. G. Doettinger et al., Phys. Rev. Lett. 73, 1691 (1994).

[10] Z. L. Xiao et al., Phys. Rev. B 53, 15265 (1996).

[11] G. M. Eliashberg, JETP Lett. 11, 114 (1970) [Sov. Phys. JETP 34, 668 (1972)]; and B. I. Ivlev, S. G. Lisitsyn, and G. M. Eliashberg, J. Low Temp. Phys. 10, 449 (1973).

[12] A. I. Bezuglyj and V. A. Shklovskij, Physica C 202, 234 (1992).

[13] A. I. Bezuglyj, Physica C 323, 122 (1999).

[14] V. G. Voltskaya, et al., Sov. J. Low Temp. Phys. 18, 683 (1993).

[15] S. G. Doettinger et al., Physica C 251, 285 (1995).

[16] H. Nakagawa, N. Miura, and Y. Enomoto, J. Phys.: Condens. Matter 10, 11571 (1998).

[17] N. R. Werthamer, E. Helfand, and P. C. Hohenberg, Phys. Rev. 147, 295 (1966).

[18] W. Lanping et al., Phys. Rev. B 40, 10954 (1989); and R. T. Collins et al., Phys. Rev. Lett. 59, 704 (1987).

[19] C. Poole, H. A. Farach, and R. J. Creswick, Superconductivity (Academic Press, San Diego, 1995).

[20] M. N. Kunchur, D. K. Christen, C. E. Klabunde, and J. M. Phillips, Phys. Rev. Lett. 72, 752 (1994).

[21] M. N. Kunchur, Mod. Phys. Lett. B. 9, 399 (1995).

[22] NIST WebHTS Database, http://www.ceramics.nist.gov/srd/hts/htsquery.htm

[23] C. Kittel, Introduction to Solid State Physics, seventh edition, (John Wiley and Sons, New York, 1996), appendix J.

[24] The author has an ongoing project (funded by Lockheed Martin, Inc.) to explore the high-dissipation regime for the operation of pulsed superconductive magnets for fusion energy devices. 